\title{
Atitude para o consumo colaborativo: um estudo baseado na consciência ambiental
}

\section{Attitude for collaborative consumption: a study based on environmental awareness}

\author{
Luis Eduardo Brandão Paiva ${ }^{1}$ \\ Emanuella Lustosa Bandeira² \\ Hanna Rocha de Arruda $^{3}$ \\ Cláudia Buhamra Abreu Romero ${ }^{4}$
}

\section{Resumo}

Diante da preocupação com o desenvolvimento sustentável, temáticas sobre consumo colaborativo e consciência ambiental alinham-se para reduzir os descartes e subprodutos emitidos ao meio ambiente. Sendo assim, esta pesquisa tem como objetivo analisar a influência da consciência ambiental na atitude para o consumo colaborativo de compra, venda e troca em plataformas digitais. Trata-se de uma pesquisa quantitativa, realizada com 125 universitários do curso de Administração. Utiliza-se estatística descritiva e inferencial, análise fatorial exploratória e regressão logística para caracterização sociodemográfica dos usuários, bem como para a análise da atitude para o consumo colaborativo e das dimensões da consciência ambiental: consumo engajado, preocupação com o lixo, mobilização e ambiente doméstico. Os resultados indicam que os universitários conhecem o conceito de consumo colaborativo e que, quanto mais familiarizados com essa temática, maior a frequência e o tempo médio de uso das plataformas digitais, sendo as mais operadas OLX e Mercado Livre, principalmente para fins de redução de custos e do tempo de transação, prevalecendo benefícios individuais. Ademais, verificam-se influências positivas da preocupação com o lixo e da renda individual na atitude para o consumo colaborativo, e influências negativas da maturidade dos estudantes, e da média de uso das plataformas digitais na atitude para o consumo colaborativo. Sugere-se, portanto, o desenvolvimento de políticas públicas que estimulem a consciência ambiental para fomentar o consumo colaborativo mais sustentável, bem como a realização de pesquisas qualitativas junto a empresas de consumo ambiental para aprofundar temáticas emergentes, como a preocupação com o lixo.

Palavras-chave: Consumo Colaborativo; Consciência Ambiental; Plataformas digitais.

\section{Abstract}

In sight of the concern with sustainable development, themes on collaborative consumption and environmental awareness are aligned to reduce the discards and by-products emitted to the environment. Therefore, this research aims to analyze the influence of environmental

\footnotetext{
${ }^{1}$ Doutorando em Administração e Controladoria na Universidade Federal do Ceará (UFC).

${ }^{2}$ Doutoranda em Administração e Controladoria na UFC.

${ }^{3}$ Doutoranda em Administração e Controladoria na UFC.

${ }^{4}$ Doutora em Administração, Docente no Programa de Pós-Graduação em Administração e Controladoria da UFC.
} 
awareness on the attitude towards collaborative consumption of buying, selling and exchanging on digital platforms. It is a quantitative research, carried out with 125 university students in the Business Administration course. Descriptive and inferential statistics, exploratory factor analysis and logistic regression are used for sociodemographic characterization of users, as well as for the analysis of the attitude towards collaborative consumption and the dimensions of environmental awareness: engaged consumption, concern with waste, mobilization and home environment. The results indicate that university students know the concept of collaborative consumption and that, the more familiar with this theme, the greater the frequency and average time of using digital platforms, the most operated are OLX and Mercado Livre, mainly for the purpose of cost reduction and reduction of transaction time, with individual benefits prevailing. In addition, there are positive influences of the concern with garbage and individual income in the attitude towards collaborative consumption, and negative influences on the maturity of students and the average use of digital platforms in the attitude towards collaborative consumption. It is suggested, therefore, the development of public policies that support environmental awareness to encourage more sustainable collaborative consumption, as well as the conduct of qualitative research with environmental consumption companies to deepen emerging themes, such as the concern with waste.

Keywords: Collaborative consumption. Environmental awareness. Online platforms.

\section{Introdução}

A problemática do século XXI traz questões relacionadas ao desenvolvimento sustentável, modelo que prevê a integração entre economia, sociedade e meio ambiente, de modo que o crescimento econômico pode-se basear na inclusão social e na proteção ambiental (Rio+20, 2017; Young \& Tilley, 2006). Nesse sentido, a décima segunda meta, definida pela ONU para o desenvolvimento sustentável, refere-se ao consumo e à produção responsável, cujo objetivo é fazer mais e melhor com menos (Thakur, 2016). Para alcançá-la, entre outros aspectos, é discutida a redução de insumos, da degradação e da poluição, bem como o prolongamento do ciclo de vida dos produtos, que podem promover melhorias na qualidade de vida da população (Gmelin \& Seuring, 2014; Hult, 2011). Diante da polissemia do termo desenvolvimento sustentável, faz-se necessária a conscientização da população sobre a importância do empenho pessoal nesse complexo processo de consumo ecologicamente consciente (Griggs et al., 2013).

Uma das possibilidades de alcançar a redução de recursos e o aumento da utilidade dos produtos é por meio do consumo colaborativo, que inclui o compartilhamento, a troca e o aluguel de bens tangíveis (Benjaafar, Kong, \& Courcoubetis, 2018) por meio de plataformas digitais. Essa modalidade de consumo pode possibilitar que uma maior quantidade de pessoas utilize um bem, aumentando, consequentemente, o seu ciclo de vida e o seu acesso com menor custo. Esse modelo requer pouco ônus ao indivíduo em comparação com a economia 
tradicional e utiliza plataformas digitais como intermediárias do consumo (Belk, 2014; Botsman \& Rogers, 2011; Habibi, Davidson, \& Laroche, 2017; Ornellas, 2013).

Estudos indicam que a principal motivação para a realização do consumo colaborativo é o benefício econômico, apesar disso, as questões ambientais também se mostram como razões significativas (Arruda, Bandeira, Silva, \& Rebouças, 2016; Botsman \& Rogers, 2011; Hamari, Sjöklint, \& Ukkonen, 2016). Uma pesquisa realizada em 2015 pelo instituto Market Analysis revelou que estar familiarizado com o conceito de consumo colaborativo pode aumentar a prática deste, afinal os achados dessa pesquisa indicaram que pelo menos um em cada três familiarizados praticou alguma forma de consumo compartilhado nos últimos 12 meses (Market Analysis, 2015).

Iniciativas inspiradoras surgidas nos últimos anos em plataformas digitais (FazoQuê, Tem Açúcar?, Zipcar, Airbnb, Couchsurfing) mostram uma nova perspectiva de consumo sustentável e consciente (EcoD, 2016; Zervas, Proserpio, \& Byers, 2017). Os mercados de redistribuição, como as plataformas digitais, facilitam operações de compra, venda e troca, oportunizando o acesso de compradores e trocadores interessados em bens sem utilidade aos seus antigos donos, bem como a venda de produtos em desuso, aumentando assim o tempo de utilização dos objetos (Botsman \& Rogers, 2011).

Além disso, o Research Designed for Strategy (RDS) aponta que jovens bem instruídos podem ser considerados os principais usuários do consumo colaborativo (RDS, 2016), como é o caso dos universitários, que podem compreender com mais facilidade questões relacionadas com a proteção e conservação do meio ambiente, sendo mais propensos ao engajamento com as questões ambientais e com o consumo colaborativo, quando comparados com pessoas menos instruídas (Han \& Kim, 2010; Hedlund, 2011; Paço, Ferreira, Raposo, Rodrigues, \& Dinis, 2011). Entretanto, não se descarta aqui a consciência ambiental desenvolvida, muitas vezes, por conta do ofício, sendo o caso de catadores de recicláveis e de lixo, garis, entre outros profissionais que impactam de forma direta ou indireta no meio ambiente.

Diante da relação entre a consciência ambiental e o consumo colaborativo, surge o seguinte questionamento: qual a influência da consciência ambiental na atitude para o consumo colaborativo dos universitários? Esta pesquisa tem como objetivo, portanto, analisar a influência da consciência ambiental na atitude para o consumo colaborativo de compra, venda e troca em plataformas digitais. Adicionalmente, pretende-se caracterizar o perfil sociodemográfico desses usuários. 
Espera-se que a presente pesquisa possa contribuir com o conhecimento no campo do consumo colaborativo, sobretudo por alinhá-lo com a consciência ambiental, de modo a ampliar e evoluir com abordagens empíricas sobre os temas em questão. Além disso, os resultados podem mostrar aos potenciais investidores do consumo colaborativo, as possíveis conexões e os impactos da consciência ambiental nos negócios, orientando a tomada de decisão dos gestores, e, ainda, subsidiando a implementação de políticas ambientais e colaborativas que possam estimular a prática do consumo colaborativo e a consolidação de negócios mais sustentáveis.

Além desta introdução, o artigo está segmentado em mais cinco seções. Na segunda, apresenta-se a fundamentação teórica da pesquisa com discussões sobre consciência ambiental e consumo colaborativo, que serviram de base para o desenvolvimento da hipótese do estudo. Na terceira, abordam-se os procedimentos metodológicos utilizados para a obtenção dos resultados. Na quarta, tem-se a análise e discussão dos resultados. E na quinta, destaca-se a conclusão da pesquisa com reflexões para estudos futuros sobre consumo colaborativo e consciência ambiental.

\section{Fundamentação teórica}

\subsection{Consciência ambiental}

Os problemas relacionados ao meio ambiente estão servindo de alerta a pesquisas que buscam compreender causas e efeitos da proteção ou da conservação ambiental (Pato \& Tamayo, 2006). Diante disso, as investigações sobre o campo do conhecimento relacionado ao meio ambiente, considerando a construção da consciência ambiental, emergem a partir da década de 1970, com o intuito de compreender o comportamento sustentável do indivíduo (Kinnear, Taylor, \& Ahmed, 1974; Oskamp, 2000; Verdugo, 2012). Verifica-se que esses estudos são relativamente recentes, desafiando pesquisadores por meio de enfoques diversos e buscando contribuir para o campo multidisciplinar dessa área do conhecimento científico (Hutchison, 2018; Pato \& Tamayo, 2006; Paiva, Lima, Sousa, \& Pontes, 2019).

Schlegelmilch, Bohlen e Diamantopoulos (1996) definem consciência ambiental como um construto multidimensional, composto por elementos cognitivos, atitudinais e comportamentais. Klineberg, McKeever e Rothenbach (1998) apontam que se pode mensurar o nível de consciência ambiental de quatro formas: (i) fornecendo opções entre proteção ambiental e interesses políticos e econômicos futuros, como aumento na taxa de emprego e 
crescimento econômico; (ii) fazendo questionamentos a respeito da percepção dos indivíduos sobre a poluição do meio ambiente; (iii) descobrindo o nível de engajamento com atividades em prol do meio ambiente; e (iv) fazendo perguntas abstratas sobre danos globais ao meio ambiente.

Bedante e Slongo (2004) definem consciência ambiental como a tendência de um indivíduo tratar os assuntos relativos ao meio ambiente de uma maneira favorável ou desfavorável. Logo, indivíduos com maiores níveis de consciência ambiental estariam mais propensos ao processo de tomada de decisão levando em consideração seus impactos ambientais. Pato e Tamayo (2006) entendem a consciência ambiental como o comportamento ambiental do indivíduo diante da proteção ou conservação do meio ambiente.

Os estudos empíricos sobre a mensuração da consciência ambiental emanam, sobretudo, a partir das medidas de Karp (1996) e Kaiser, Ranney, Hartig e Bowler (1999) que buscam mensurar o nível de consciência ambiental. Nesse contexto, pesquisas sobre esse constructo são caracterizadas, em sua maioria, pela observação, investigação e compreensão de ações individuais que possam impactar o meio ambiente (Kaiser, Ranney, Hartig, \& Bowler, 1999; Karahan \& Roehrig, 2015; Verdugo, 2012).

Schmuck e Schultz (2002), Bedante e Slongo (2004), Gonçalves-Dias, Teodósio, Carvalho e Silva (2009), Verdugo (2012) e Morrison e Beer (2017) abordam a consciência ambiental como fundamental para investigar atitudes, intenções e comportamentos de indivíduos no meio ambiente. Nessa perspectiva, alguns estudos estão voltados para comportamentos ambientais mais específicos, por exemplo: conservação de água (Lam, 1999), reciclagem (Vining \& Ebreo, 1992) e energia (Silva, Cunto, \& Guevara, 2016).

Diante das especificidades e das peculiaridades de estudos que versam sobre o meio ambiente, com base nos estudos de Karp (1996), Kaiser et al. (1999), Straughan e Roberts (1999) e Pato e Tamayo (2006); Gonçalves-Dias et al. (2009) propuseram um instrumento para mensurar a consciência ambiental dos universitários do curso de Administração, sendo composto por cinco dimensões, a saber: consumo engajado; preocupação com o lixo; boicote via consumo; mobilização; e ambiente doméstico (Figura 1).

\begin{tabular}{l|l}
\hline Aspectos & Descrição \\
\hline Consumo & $\begin{array}{l}\text { Agrupa variáveis referentes às atitudes dos respondentes quanto ao consumo. } \\
\text { Exprejado }\end{array}$ \\
$\begin{array}{l}\text { Exvolvem a postura dos fabricantes e um caráter mais ativo na procura de opções de } \\
\text { produtos ecologicamente corretos. }\end{array}$ \\
\hline $\begin{array}{l}\text { Preocupação com o } \\
\text { Lixo }\end{array}$ & $\begin{array}{l}\text { Reúne variáveis ligadas à atitude dos indivíduos quanto ao lixo e à limpeza de } \\
\text { ambientes domésticos e públicos. }\end{array}$ \\
\hline
\end{tabular}




\begin{tabular}{l|l}
\hline $\begin{array}{l}\text { Boicote via } \\
\text { Consumo }\end{array}$ & $\begin{array}{l}\text { Aglutina variáveis comportamentais relacionadas ao consumo. Contudo, o caráter da } \\
\text { postura dos indivíduos indica maior propensão a penalizar produtos e serviços } \\
\text { ecologicamente incorretos. }\end{array}$ \\
\hline Mobilização & $\begin{array}{l}\text { Agrega variáveis comportamentais relacionadas a uma postura proativa na busca da } \\
\text { sensibilização de outros indivíduos no que se refere às questões ambientais. }\end{array}$ \\
\hline $\begin{array}{l}\text { Ambiente } \\
\text { Doméstico }\end{array}$ & $\begin{array}{l}\text { Agrupa variáveis ligadas ao comportamento do indivíduo na vida domiciliar. As } \\
\text { variáveis estão relacionadas ao uso cotidiano de recursos naturais, como energia } \\
\text { elétrica e água. }\end{array}$ \\
\hline
\end{tabular}

Figura 1. Dimensões da consciência ambiental

Fonte: Adaptado de Gonçalves-Dias et al. (2009).

Nessa perspectiva, a consciência ambiental é investigada por meio da preocupação do indivíduo com as dimensões ambientais, as quais beneficiam e podem impactar o meio ambiente e a sociedade como um todo (Giudici, Guerini, \& Rossi-Lamastra, 2019; GonçalvesDias, Teodósio, Carvalho, \& Silva, 2009; Kuckertz \& Wagner, 2010). Os impactos positivos das ações dos indivíduos no meio ambiente podem trazer soluções que reduzam a degradação e o desgaste de produtos no meio ambiente, contribuindo diretamente para ampliar a proteção ambiental e as práticas de consumo responsável, de forma mais colaborativa e sustentável.

\subsection{Consumo colaborativo}

Doin (2019) indica alguns desafios na compreensão da economia do compartilhamento, destacando: o fato da literatura apresentar rótulos e definições distintos, ora sobrepostos ora dissociados; as variadas formas que os distintos termos são utilizados no campo teórico e prático, e a limitação do escopo da economia do compartilhamento (Gerwe \& Silva, 2020) que pode incluir ou não transações monetárias. Essas divergências e dificuldades de delimitação ocorrem tanto no âmbito nacional como internacional (Benjaafar et al., 2018; Habibi, Kim, \& Laroche, 2016; Rivera, Gordo, Cassidy, \& Apesteguía, 2017). No Brasil, mesmo em um segmento mais jovem e informado, o conceito ainda não está bem difundido, conforme indicado em algumas pesquisas (RDS, 2016).

A ideia do compartilhamento-economia íntima surge inicialmente na literatura por meio de Price (1975, p. 4) que o definiu como "economia íntima [familiar] [...] é a alocação de bens e serviços econômicos sem calcular os retornos, que é [...] tanto emocional quanto racional, com baixa formalidade e baixa necessidade de reconhecer o que é tomado e dado". Posteriormente, Felson e Spaeth (1978) definem de forma pioneira o consumo colaborativo como eventos em que as pessoas consomem bens ou serviços econômicos no processo de engajar-se em atividades conjuntas.

Apesar dessas iniciativas, Doin (2019) ressalta que a definição mais difundida na 
mídia e na literatura como um novo modelo econômico foi a de consumo colaborativo, proposta por Botsman e Rogers (2011) que se refere diretamente ao compartilhamento tradicional, escambo, empréstimo, negociação, locação, doação e troca, redefinido por meio da tecnologia e de comunidades entre pares. Desde 2010, a maioria das pesquisas vem apresentando os termos consumo colaborativo e economia compartilhada como fenômenos comuns que sugerem a utilização do consumo com suas práticas sustentáveis, assim como da inteligência de mercado para promover uma sociedade mais colaborativa e sustentável (Heinrichs, 2013).

Nesse sentido, observa-se uma imensa variedade de práticas que se encaixam nesse conceito, desde trocas simples, que envolvem uma transferência da posse de um determinado bem material, até o serviço de compartilhamento de espaços e habilidades. Belk (2014) reforça, ainda, que as pessoas percebem vantagens na adoção do consumo colaborativo, pois geram benefícios para si e para a sociedade.

Hamari, Sjöklint e Ukkonen (2016) e Fraanje e Spaargaren (2019) enfatizam que a economia compartilhada é um fenômeno econômico-tecnológico emergente, alimentado pelas Tecnologias da Informação e da Comunicação (TIC's), ressaltando a utilização de plataformas digitais como inerentes ao consumo colaborativo. A popularidade das TIC's promove a ampliação das formas tradicionais de troca, assumindo novos formatos e modificando a natureza do consumo, do marketing e da sociedade (Castro, Moura, Cunha, \& Pires, 2016).

O consumo colaborativo não só surgiu, mas teve a sua rápida explosão nas formas tradicionais de compartilhamento, troca, empréstimo e locação por meio de tecnologias de rede em grande escala (Botsman \& Rogers, 2011; Maurer, Schmitt, Campos, Silva, \& Barcellos, 2015; Ornellas, 2013). A internet, fruto da sociedade moderna e a mesma que desperta no homem o individualismo e a cultura do "eu", de forma paradoxal, vem aproximando pessoas, desconstruindo e reconstruindo algumas formas de consumo, inclusive, favorecendo o surgimento de grupos de consumo colaborativo (Barcellos \& Silva, 2014). Desse modo, para Tomas, Meschgrahw e Alcantara (2012) e Hajli (2015), a internet, de modo especial a web 2.0, que permite aos usuários contribuir com conteúdo e conectar-se uns com os outros, influencia diretamente no comportamento do consumidor.

Apesar dessa visão positiva acerca dos benefícios advindos do consumo colaborativo para o desenvolvimento sustentável, é importante destacar que muitos negócios na economia colaborativa são vistos como subversivos em relação aos sistemas regulamentares e fiscais (CNMC, 2016). Indica-se que a falta de regulamentação desses novos negócios pode gerar o 
não pagamento de impostos, a possível concorrência desleal e até a exploração de mão de obra, já que muitos ofertantes não têm as obrigações fiscais e legais que outras empresas do mesmo segmento (Belk, 2014; Schor, 2014).

Com base nessas abordagens, vale destacar como exemplo o compartilhamento gratuito de música digital e filmes que levou a indústria da música e do cinema a perder vendas substanciais de CDs e DVDs, levando-os a se envolverem em uma série de ações que tentam impor seus direitos de propriedade intelectual. A "guerra ao compartilhamento" resultante (Aigrain, 2012) provou ser amplamente inútil, pois os sites alternativos de compartilhamento surgem em proporções geométricas (Belk, 2014). Além disso, a forma com que as pessoas utilizam o valor monetário recebido das transações colaborativas é questionável do ponto de vista ambiental, afinal esse recurso pode estar sendo usado para comprar produtos não sustentáveis (Habibi et al., 2017; Martin, 2016).

Nesse contexto, a economia colaborativa ainda traz uma série de questões a serem discutidas. Morozov (2013) a critica duramente afirmando que é uma forma de "neoliberalismo em esteroides" por comercializarem aspectos da vida que antes estavam distantes do mercado. Martin (2016) e Habibi, Davidson e Laroche (2017) complementam que o fluxo entre a demanda e a oferta no consumo colaborativo pode criar uma economia de mercado que vem apresentando grande crescimento, dividindo espaço com a economia tradicional.

Arruda, Bandeira, Silva e Rebouças (2016) ressaltam que a economia colaborativa apresenta diversos benefícios individuais (economia de custos, viabilidade de acessar bens que não teria condições de possuir) e sociais (extensão do ciclo de vida dos produtos, diminuição do uso de matéria-prima, redução do lixo). Freitas, Petrini e Silveira (2016) reiteram que o consumo colaborativo pode impactar diretamente os hábitos, as atitudes, as práticas e a consciência ambiental das pessoas, seja no âmbito econômico seja no social ou ambiental.

Apesar das divergências típicas, o consumo colaborativo e a consciência ambiental tornaram-se fenômenos sociais e econômicos de destaque em poucos anos (Gonçalves-Dias et al., 2009; Rivera et al., 2017). Dessa forma, a consciência das pessoas que têm atitudes positivas em relação ao consumo colaborativo pode impactar diretamente no meio ambiente. Com base nas discussões abordadas, destaca-se, a seguir, o desenvolvimento da hipótese da pesquisa, a qual busca elucidar a interconexão entre o consumo colaborativo e a consciência ambiental. 


\subsection{Desenvolvimento da hipótese}

$\mathrm{Na}$ busca pela compreensão do comportamento do consumidor brasileiro frente à Economia Colaborativa, o Research Designed For Strategy (2016) ressaltou que pouco mais da metade dos brasileiros tem alguma referência ou informação sobre economia colaborativa, sendo essa quantidade maior entre os consumidores mais qualificados em termos econômicos, culturais e de acesso à tecnologia. Além disso, os indivíduos que têm mais conhecimento sobre economia colaborativa também apresentaram maiores preocupações com o consumo consciente e com as causas relacionadas à sustentabilidade, tanto pelos impactos de suas ações individuais como pelas ações coletivas.

Com base no contexto ambiental que vem, nas duas últimas décadas, permitindo reflexões acerca do consumo, evidenciam-se estudos que investigam o impacto da consciência dos consumidores frente aos problemas ambientais, assim como o descarte de produtos que possam degradar o meio ambiente (Deus, Afonso, \& Afonso, 2014; Han \& Kim, 2010; Vermeir \& Verbeke, 2008). Quanto à utilização dos recursos naturais, a preocupação com o planeta e com as pessoas intensificam discussões e debates sobre as questões ambientais e o consumo (Kinnear et al., 1974; Morrison \& Beer, 2017).

Bedante e Slongo (2004) afirmam que o agravamento dos problemas ambientais ocorridos nos últimos cem anos pode estar diretamente associado ao uso indiscriminado dos recursos existentes no meio ambiente. Como resposta a esse cenário, surgiram movimentos ambientalistas e um novo tipo de consumidor - o consumidor ecologicamente consciente - o qual passou a ponderar os impactos de seu padrão de consumo na natureza. Nesse contexto, alguns estudos vêm sendo conduzidos na tentativa de compreender a influência exercida pela consciência ambiental no consumo sustentável.

Diante disso, o consumo colaborativo pode-se alinhar diretamente com a temática ambiental, sobretudo por meio da consciência ambiental do indivíduo (Freitas, Petrini, \& Silveira, 2016), pois esta fomenta a compra, a venda e a troca de produtos usados, assim como o compartilhamento e os aluguéis, por curto prazo, de produtos e serviços, maximizando a utilidade destes, e reduzindo os subprodutos e os descartes no meio ambiente. A consciência ambiental propicia a aquisição de comportamentos, posturas e ações que podem impactar a conduta dos indivíduos no meio ambiente (Afonso, Zanon, Locatelli, \& Afonso, 2016; Gonçalves-Dias et al., 2009; Karahan \& Roehrig, 2015; Morrison \& Beer, 2017).

Verdugo (2012) e Morrison e Beer (2017) consideram a consciência ambiental um fator propulsor do comportamento ambientalmente favorável. Nesse contexto, o consumo 
colaborativo pode ser apresentado como uma alternativa viável aos indivíduos que se mostram conscientes do impacto de seu consumo no meio ambiente (Jaeger-Erben, RückertJohn, \& Schäfer, 2015) e que, ao mesmo tempo, não querem deixar de consumir bens e serviços que atendam suas necessidades individuais.

Leismann, Schmitt, Rohn e Baedeker (2013) destacam a importância das inovações tecnológicas para as novas perspectivas de consumo que abordam aspectos sustentáveis das novas práticas. Esses autores identificaram também que essas inovações, como internet e suas plataformas, se somadas a novas formas comerciais do uso em vez de posse, são capazes de levar a uma redução substancial no consumo de recursos naturais.

Diante das discussões abordadas e com o intuito de fortalecer e alinhar a consciência ambiental e as atitudes das pessoas para o consumo colaborativo, sobretudo por meio da compra, venda ou troca de produtos usados em plataformas digitais, evidencia-se a seguinte hipótese para esta pesquisa:

H1: Há influência positiva da consciência ambiental na atitude para o consumo colaborativo.

\section{Procedimentos metodológicos}

Trata-se de uma pesquisa de natureza quantitativa, descritiva, de recorte temporal transversal, com utilização do método survey para a coleta dos dados. A população do estudo é composta por universitários do curso de Administração de uma Universidade Pública do estado do Ceará, considerada a maior do estado e, ainda, no ranking das 32 universidades brasileiras listadas como as melhores da América Latina, de acordo com a tradicional publicação britânica Times Higher Education (THE).

Foram investigados universitários que realizam compra, venda ou troca de produtos usados por meio de plataformas digitais amplamente utilizadas no Brasil (Mercado Livre, OLX, Grupos do Facebook, Enjoei). Na Universidade investigada, existem aproximadamente 900 estudantes no curso de Administração, sendo obtida uma amostra de 125 universitários, o que representa aproximadamente $14 \%$ da população. A escolha de universitários está associada ao perfil etário e educacional, fazendo com que esse público possa estar mais inclinado à utilização de plataformas digitais para o consumo colaborativo.

Por alinhamento com o estudo de Gonçalves-Dias et al. (2009), optou-se também pela delimitação do curso de Administração. Esses autores esclarecem que a formação de administradores é um dos campos da educação nos quais os desafios de mudança do 
comportamento ambiental se apresentam de maneira mais decisiva. Afinal, muitos dos egressos desse curso podem ocupar cargos estratégicos nas organizações, influenciando na criação e implementação de modelos de gestão favoráveis (ou não) ao meio ambiente. Han e Kim (2010) e Paço, Ferreira, Raposo, Rodrigues e Dinis (2011) reiteram que, pelo fato de o curso de Administração estar ligado à gestão de empresas, os estudantes podem estar mais atentos às questões ambientais e de consumo e, consequentemente, engajar-se mais com essas causas.

A amostra por conveniência é composta exclusivamente por alunos matriculados na modalidade presencial. Tal escolha justifica-se pela facilidade de acesso aos participantes. O método adotado foi o não-probabilístico, pois, de acordo com Hair, Black, Babin, Anderson e Tatham (2009), pode ter probabilidade desconhecida e tem base na intuição e no conhecimento dos pesquisadores.

O instrumento de coleta de dados utilizado é composto por duas escalas adaptadas de Gonçalves-Dias et al. (2009) e Hamari et al. (2016), as quais buscam mensurar a consciência ambiental e a atitude do consumo colaborativo, respectivamente. Para verificar a clareza dos itens, bem como o tempo de aplicação do questionário, realizou-se um pré-teste com 40 respondentes. Verificou-se, ainda, que o tempo médio de resposta é de 9,5 minutos. A coleta dos dados foi realizada entre os meses de outubro e novembro de 2017 , de forma presencial, em que os pesquisadores foram diretamente nas salas de aula para a obtenção dos dados.

O questionário adaptado é composto por 41 questões, sendo segmentado em três blocos: consciência ambiental (16 itens), consumo colaborativo (9 itens) e perfil do consumidor (16 itens). No primeiro bloco, consciência ambiental, os itens foram mensurados de uma escala diferencial semântica de 5 pontos $(1=$ Nunca e $5=$ Sempre $)$ e divididos em cinco dimensões: consumo engajado, preocupação com o lixo, boicote via consumo, mobilização, ambiente doméstico. Em relação ao segundo bloco, consumo colaborativo, os itens mensuraram a atitude quanto ao consumo colaborativo, representado neste artigo pelas atividades de compra, venda ou troca em plataformas digitais, e foram mensurados a partir de escala Likert de concordância de cinco pontos $(1=$ discordo totalmente e $5=$ concordo totalmente).

Foram utilizadas, além da estatística descritiva e inferencial, a análise fatorial exploratória, visto que possibilita a identificação dos fatores da consciência ambiental e do consumo colaborativo. Para tal análise, usa-se o critério da variável substituta, isto é, utilizase a variável com o maior poder de explicação dentro de cada fator em modelos de regressão. Por fim, faz-se o uso do modelo da regressão logística com o intuito de verificar a influência 
da consciência ambiental, bem como das variáveis sociodemográficas na atitude para o consumo colaborativo, verificando-se as variáveis estatisticamente significantes na análise (nível de significância $<0,05$ ). Para o tratamento dos dados, utilizou-se o software Statistical Package for the Social Sciences (SPSS) (versão 22.0).

\section{Análise e discussão dos resultados}

\subsection{Perfil da amostra}

A amostra coletada $(\mathrm{N}=125)$ representa os universitários do curso de Administração de uma Universidade Pública, mostrando-se, então, as seguintes características: predomínio de solteiros $(84,8 \%)$ e homens (55\%); idade média de 24 anos, variando entre 17 e 60 anos; renda pessoal de até $\mathrm{R} \$ 1.500,00(58,4 \%)$ e familiar acima de $\mathrm{R} \$ 7.000,00(30,4 \%)$.

Em relação às plataformas digitais, verifica-se que a principal finalidade de utilização é a compra de produtos usados (64,8\%); as mais operadas são OLX $(39,2 \%)$ e Mercado Livre (29,6\%); utilizadas acerca de 1 a 2 anos (30,4\%); a frequência média de uso é semestral (46,4\%); e a maior parte das transações acontece nas categorias: eletrônicos (39,2\%) e livros (20\%) (Tabela 1).

Tabela 1.

\section{Categorias de produtos usados}

\begin{tabular}{lcc}
\hline \multicolumn{1}{c}{ Categorias } & $(\mathrm{N}=125)$ & $(\%)$ \\
\hline Eletrônicos & 49 & 39,2 \\
Livros & 25 & 20,0 \\
Calçados, roupas ou bolsas & 22 & 17,6 \\
Casa, móveis e decoração & 10 & 8,0 \\
Outros & 6 & 4,8 \\
Relógios, joias, bijuterias ou acessórios & 5 & 4,0 \\
Eletrodomésticos & 4 & 3,2 \\
Carro, moto ou acessórios automotivos & 3 & 2,4 \\
Saúde e beleza & 1 & 0,8 \\
\hline
\end{tabular}

Fonte: Dados da pesquisa.

A despeito das motivações para a utilização das plataformas digitais, solicitou-se que os respondentes indicassem, de forma hierárquica, as três principais razões para o usá-las, sendo essas, respectivamente, redução de custos $(74,4 \%)$, redução do tempo de transação (44\%) e conveniência e facilidade (29,6\%). Ressalta-se, entretanto, que o respondente poderia marcar mais de uma motivação para a utilização dessas plataformas (múltiplas respostas), por 
isso que o percentual ultrapassa os 100\% (Tabela 2).

Tabela 2.

Motivações para a utilização das plataformas online

\begin{tabular}{lcc}
\hline \multicolumn{1}{c}{ Motivações } & $(\mathrm{N}=125)$ & $(\%)$ \\
\hline Reduzir custos & 93 & 74,4 \\
Reduzir tempo de transação & 55 & 44,0 \\
Ter conveniência e facilidade & 37 & 29,6 \\
Obter uma renda extra & 27 & 21,6 \\
Evitar o consumo excessivo & 22 & 17,6 \\
Sentir segurança nas transações realizadas & 18 & 14,4 \\
Consumir de forma sustentável & 18 & 14,4 \\
Evitar o acúmulo excessivo & 15 & 12,0 \\
\hline
\end{tabular}

Fonte: Dados da pesquisa.

Em contrapartida, as motivações menos recorrentes são sentir segurança nas transações realizadas $(14,4 \%)$, consumir de forma sustentável $(14,4 \%)$ e evitar o acúmulo excessivo (12\%). Esses achados corroboram com o enfatizado por Arruda et al. (2016), pois foram identificados benefícios individuais, como economia de custos e tempo, sendo estes mais importantes do que os coletivos, como o consumo consciente e o bem-estar da sociedade, e isto reforça o uso dessas plataformas como uma forma de redução de custos (Barnes \& Mattsson, 2016; Benjaafar et al., 2018). Tais resultados também vão ao encontro dos benefícios indicados por Maurer, Schmitt, Campos, Silva e Barcellos (2015), os quais observaram, ao investigarem gestores de organizações e usuários de distintos sistemas de consumo colaborativo, que os usuários citaram com maior frequência os benefícios econômicos, sociais e de conveniência. Questões de confiança e sustentabilidade também aparecem nos resultados, porém com menos frequência.

Em relação ao consumo colaborativo, conforme indicado na Tabela 3 (em que o respondente também poderia marcar mais de uma alternativa), foi observado que 56 universitários conhecem o conceito e, além da compra, venda ou troca de produtos usados em plataformas digitais, também realizam outras formas de consumo colaborativo, sendo predominante o uso de bicicletas compartilhadas $(44,8 \%)$, o que corrobora com os achados das pesquisas de Patricio e Kruszielski (2016) e El-Assi, Mahmoud e Habib (2017).

Tabela 3.

Formas de consumo colaborativo

\begin{tabular}{lcc}
\hline \multicolumn{1}{c}{ Formas de consumo colaborativo } & $(\mathrm{N}=125)$ & $(\%)$ \\
\hline Bicicletas compartilhadas (bikesharing) & 56 & 44,8 \\
Não realizo outras formas de consumo & 55 & 44,0 \\
Imóveis compartilhados (exemplos: airbnb, couchsurfing) & 17 & 13,6
\end{tabular}


Fonte: Dados da pesquisa.

Diante da realização de uma tabulação cruzada entre o conhecimento do conceito de consumo colaborativo e as motivações para compra, venda ou troca de produtos usados nas plataformas digitais, constatou-se que não há diferença estatisticamente significante entre os universitários que conhecem ou não esse conceito. Ou seja, quem demonstrou estar familiarizado com essa temática não revelou motivações distintas de quem não está familiarizado, e isto permite inferir que, para a modalidade de consumo colaborativo pesquisada, prevalecem razões relacionadas à redução de custo e tempo em detrimento daqueles associados ao consumo sustentável.

Ademais, analisou-se a relação cruzada entre o conhecimento do conceito de consumo colaborativo e a frequência de uso das plataformas digitais, assim como entre o conhecimento do conceito de consumo colaborativo e o tempo médio de uso das plataformas digitais. Identificou-se que quanto maior a familiaridade com a temática, maior é a frequência de uso, ressaltando que os universitários que conhecem o conceito de consumo colaborativo utilizam as plataformas digitais duas vezes mais a cada mês do que os que não o conhece; e também é maior o tempo médio de uso; $44,3 \%$ dos estudantes que conhecem o conceito utilizam as plataformas digitais há pelo menos 2 anos, enquanto $67,2 \%$ dos que não o conhecem usam a menos tempo, entre 6 meses a 2 anos.

Ressalta-se a significância dessa análise por meio do valor $\mathrm{p}$ do qui-quadrado de 0,026, o que é fortemente aceitável, pois a literatura mostra que um valor abaixo de 0,05 reforça que a análise é significante. De acordo com Hair et al. (2009), o teste do qui-quadrado possibilita testar se duas ou mais amostras (ou grupos) independentes que diferem relativamente a uma determinada característica (Tabela 4).

Tabela 4.

Conhecimento de consumo colaborativo versus tempo médio de uso das plataformas online

\begin{tabular}{|c|c|c|c|c|}
\hline \multirow[b]{2}{*}{ Tempo de utilização das plataformas } & \multicolumn{2}{|c|}{ Consumo colaborativo } & \multirow[b]{2}{*}{ Total } & \multirow{2}{*}{$\begin{array}{c}\text { Valor p do } \\
\text { qui- } \\
\text { quadrado }\end{array}$} \\
\hline & Sim & Não & & \\
\hline \multirow[t]{2}{*}{ Menos de 6 meses } & 4 & 8 & 12 & \multirow{8}{*}{,026 } \\
\hline & $5,7 \%$ & $14,5 \%$ & $9,6 \%$ & \\
\hline \multirow[t]{2}{*}{ De 6 meses a 1 ano } & 16 & 18 & 34 & \\
\hline & $22,9 \%$ & $32,7 \%$ & $27,2 \%$ & \\
\hline \multirow[t]{2}{*}{ De 1 ano a 2 anos } & 19 & 19 & 38 & \\
\hline & $27,1 \%$ & $34,5 \%$ & $30,4 \%$ & \\
\hline \multirow[t]{2}{*}{ De 2 anos a 3 anos } & 17 & 4 & 21 & \\
\hline & $24,3 \%$ & $7,3 \%$ & $16,8 \%$ & \\
\hline
\end{tabular}




\begin{tabular}{lccc}
\hline Mais de 3 anos & 14 & 6 & 20 \\
& $20,0 \%$ & $10,9 \%$ & $16,0 \%$ \\
\hline Total & 70 & 55 & 125 \\
& $100,0 \%$ & $100,0 \%$ & $100,0 \%$ \\
\hline
\end{tabular}

Fonte: Dados da pesquisa.

De acordo com o achado da pesquisa realizada pelo instituto Market Analysis (2015), sobre o conhecimento de consumo colaborativo e o tempo uso das plataformas, é importante ressaltar que esse resultado converge com o desta investigação, uma vez que estar familiarizado com o conceito de consumo colaborativo pode aumentar a prática deste e que pelo menos um em cada três familiarizados praticaram alguma forma de consumo compartilhado nos últimos 12 meses.

\subsection{Análise fatorial para o consumo colaborativo e para a consciência ambiental}

Considerando as assertivas referentes ao consumo colaborativo (Hamari et al., 2016) e à consciência ambiental (Gonçalves-Dias et al., 2009), faz-se, primeiramente, o uso a Análise Fatorial Exploratória (AFE), adotando-se o critério da variável substituta para cada fator, com o uso da variável com maior carga fatorial, que permite indicar a variável que apresenta maior poder de explicação na análise (Hair, Black, Babin, Anderson, \& Tatham, 2009).

A escala de Gonçalves-Dias et al. (2009) mostrou-se satisfatória, tendo em vista que apresenta Kaiser-Meyer-Olkin (KMO) de 0,779 (>0,5) e esfericidade de Bartlett de 0,000 $(<0,1)$, indicando que esses valores estão de acordo com o patamar mínimo sugerido pela literatura (Hair et al., 2009), conforme se observa na Tabela 5.

Tabela 5.

Análise fatorial para a consciência ambiental

\begin{tabular}{lccccc}
\hline & \multicolumn{3}{c}{ Fatores } & \\
\cline { 2 - 4 } & $\begin{array}{l}\text { Consumo } \\
\text { engajado }\end{array}$ & $\begin{array}{c}\text { Ambiente } \\
\text { doméstico }\end{array}$ & $\begin{array}{c}\text { Preocupação com } \\
\text { o lixo }\end{array}$ & Mobilização & Comunalidades \\
\hline CA1 &, 750 & & & \\
CA2 &, 810 & & &, 588 \\
CA3 &, 796 & & &, 685 \\
CA4 &, $\mathbf{8 4 2}$ & & &, 664 \\
CA5 &, 538 & & &, 775 \\
CA10 &, 501 & & &, 346 \\
CA9 & &, 571 & &, 562 \\
CA14 & &, 794 & &, $\mathbf{6 4 6}$ \\
CA15 & &, 715 &, 713 &, 486 \\
CA16 & &, 648 & &, 659 \\
CA6 & & & &
\end{tabular}


Fonte: Dados da pesquisa.

Ao considerar a formação de quatro fatores (utilizando a AFE), concentrando-se nas variáveis que apresentam maiores cargas fatoriais dentro de cada fator, ressaltam-se: Consumo Engajado (CA4 "As preocupações com o meio ambiente interferem na minha decisão de compra" - carga fatorial de 0,842); Ambiente doméstico (CA14 "Tomo banho demorado" - carga fatorial de 0,794); Preocupação com o lixo (CA8 "Ajudo a manter as ruas limpas" - carga fatorial de 0,795); e Mobilização (CA12 "Mobilizo as pessoas para a conservação dos espaços públicos" - carga fatorial de 0,792).

Em relação à AFE para a atitude para o consumo colaborativo, são destacados os valores do KMO de 0,882 (>0,5) e esfericidade de Bartlett, Sig. de 0,000 $(<0,1)$, e isto possibilita apontar que a AFE para a atitude para o consumo colaborativo é satisfatória (Tabela 6).

Tabela 6.

Análise fatorial para o consumo colaborativo

\begin{tabular}{|c|c|c|}
\hline & Fator & Comunalidades \\
\hline & Atitude para o consumo colaborativo & comunaruades \\
\hline CC6 &, 715 & ,682 \\
\hline $\mathrm{CC} 7$ & ,806 & ,759 \\
\hline $\mathrm{CC8}$ & ,896 & ,881 \\
\hline CC9 & ,917 & 879 \\
\hline
\end{tabular}

Fonte: Dados da pesquisa.

Verifica-se que a variável que representa a atitude para o consumo colaborativo é: CC8 "Posso imaginar-me aumentando minhas atividades de compra, venda ou troca de produtos usados em plataformas digitais" - carga fatorial de 0,896. Diante da AFE para a consciência ambiental, bem como para a atitude para o consumo colaborativo, foram identificadas as cargas fatoriais predominantes dentro de cada fator, e isto permite indicar adequabilidade dos dados na análise fatorial, analisados mediante modelo de regressão logística. 


\subsection{Resultados do modelo de regressão logística}

A regressão logística busca verificar a relação entre uma variável dependente em função das variáveis independentes (Hair et al., 2009). Para o consumo colaborativo, adota-se como variável dependente a atitude para o consumo colaborativo que permite verificar a influência da consciência ambiental, bem como das variáveis sociodemográficas na atitude para o consumo colaborativo. Diante disso, vale reforçar que quanto menor for o sig. (grau de significância), maior o poder de influência da variável independente para predizer (ou explicar) a variável dependente. Para essa perspectiva, utiliza-se o que foi fundamentado por Hair et al. (2009), os quais sugerem que as variáveis com valores abaixo de 5\% $(0,05)$ são estatisticamente significantes e devem ser aceitas na análise (Tabela 7).

Tabela 7.

\section{Modelo de regressão}

\begin{tabular}{lccc}
\hline Variáveis & B & Sig. & Exp(B) \\
\hline Consumo engajado &, 169 &, 589 & 1,184 \\
Ambiente doméstico &, 159 &, 677 & 1,173 \\
Preocupação com o lixo & $\mathbf{1 , 3 3 9}$ & $\mathbf{, 0 1 2}$ & $\mathbf{3 , 8 1 6}$ \\
Mobilização &, 782 &, 061 & 2,186 \\
Gênero (Masculino) &, 309 &, 686 & 1,363 \\
Idade &,- 060 &, 628 &, 942 \\
Renda Individual & $\mathbf{1 , 8 9 8}$ & $\mathbf{, 0 0 2}$ & $\mathbf{6 , 6 7 3}$ \\
Renda Familiar &, 103 &, 661 & 1,108 \\
Semestre & $\mathbf{- , 3 6 7}$ & $\mathbf{, 0 2 9}$ & $\mathbf{6 9 3}$ \\
Plataforma online (OLX) &,- 769 &, 396 &, 463 \\
Plataforma online (Grupo de Facebook) &,- 990 &, 342 &, 371 \\
Finalidade Plataforma online (Venda) &, 973 &, 250 & 2,647 \\
Finalidade Plataforma online (troca) & 1,270 &, 493 & 3,562 \\
Tempo de utilização &,- 095 &, 747 &, 909 \\
Frequência de uso & $\mathbf{- 1 , 6 8 1}$ & $\mathbf{, 0 0 4}$ & $\mathbf{1 8 6}$ \\
Consumo colaborativo (não) &, 055 &, 940 & 1,057 \\
Constante &, 674 &, 879 & 1,962 \\
\hline
\end{tabular}

Fonte: Dados da pesquisa.

Em uma primeira análise, destaca-se que a preocupação com o lixo, com valores $p$ de 0,012 e B de 1,339, teve influência positiva na atitude para o consumo colaborativo. Nesse sentido, quanto maior a preocupação do indivíduo com o lixo, mais provável que a sua atitude seja favorável para o consumo colaborativo, ou seja, mais esse indivíduo pode se imaginar aumentando suas atividades de compra, venda ou troca de produtos usados em plataformas digitais. Ademais, o valor do Exp (B) de 3,816 permite inferir que esses indivíduos com 
preocupações com o lixo têm três vezes mais chances de terem atitudes para o consumo colaborativo do que os indivíduos que não têm preocupações com o lixo.

Diante dessa abordagem, percebe-se que a consciência ambiental, por meio da preocupação com o lixo, tem influência positiva na atitude para o consumo colaborativo por meio de compra, venda ou troca de produtos usados dos universitários, o que mostra fortemente o impacto da preocupação com o lixo na atitude da pessoa para o consumo colaborativo. Esse achado corrobora com as ideias de Black e Cherrier (2010), Botsman e Rogers (2011) e Maurer et al. (2015), que os indivíduos com atitudes voltadas para o consumo colaborativo podem apresentar mais preocupações ambientais com o descarte, lixo e dejetos, a e manutenção das ruas limpas. Além disso, Maurer et al. (2015), ao analisarem o discurso de usuário do consumo colaborativo, tiveram como um dos principais achados o impacto da preocupação com descarte do lixo e com o meio ambiente no consumo colaborativo dos indivíduos, e isto coincide com o que foi abordado nesta pesquisa.

Outra variável que mostrou influência positiva no tocante à atitude para o consumo colaborativo foi a renda individual, com valores p de 0,002, B de 1,898 e Exp (B) de 6,673, podendo-se ressaltar que a renda do indivíduo obteve influência positiva na atitude para o consumo colaborativo. Dessa forma, indivíduos com maiores rendas tendem a ter atitudes favoráveis quanto à prática de compra, venda ou troca de produtos usados em plataformas digitais. Os indivíduos com menores rendas estão, portanto, menos propensos a terem atitudes positivas voltadas para o consumo colaborativo. Tal achado converge com diversos estudos que ressaltam que, quanto maior a renda, maior a propensão ao consumo geral, inclusive ao consumo colaborativo (Botsman \& Rogers, 2011; Ornellas, 2013; Market Analysis, 2015).

Todavia, o semestre cursado pelos respondentes apresentou influência na atitude para o consumo colaborativo de universitários, com valores p de 0,029, B de -0,367 e Exp (B) de 0,693, e isto permitiu inferir que os universitários em semestres mais avançados, ou seja, com maior maturidade, demonstraram ter menos atitudes para o consumo colaborativo ao compará-los com os dos semestres iniciais.

Em relação à média do uso das plataformas digitais, com valores $\mathrm{p}$ de $0,004, \mathrm{~B}$ de 1,681 e Exp (B) de 0,186, os indivíduos com menos tempo de uso mostram ter atitudes mais favoráveis às práticas de compra, venda ou troca de usados em plataformas digitais do que aqueles que as utilizam há mais tempo, ou seja, ao longo do tempo de uso das plataformas, o indivíduo pode ir reduzindo a sua atitude positiva frente a tais práticas. Isso corrobora com o afirmado por Santoso e Erdaka (2015), os quais indicam que quanto maior a frequência de uso, maior a possibilidade de insatisfação, gerando atitude negativa quanto à prática de 
consumo colaborativo em plataformas digitais. Sendo assim, a experiência inicial tem um impacto positivo na fidelidade do cliente no sistema de serviço do produto.

De modo geral, os resultados encontrados, alinhados fortemente com a literatura, permitiram confirmar a hipótese desta pesquisa - $\mathbf{H}_{1}$ : Há influência positiva da consciência ambiental na atitude para o consumo colaborativo dos universitários, e isto mostra que existe uma influência da preocupação com o lixo e da renda individual, ou seja, quando maior a preocupação com o lixo e a renda individual, maior a atitude para o consumo colaborativo. Por outro lado, quando maior a frequência do uso das plataformas, menor a atitude para o consumo colaborativo, possibilitando inferir que, de certo modo, essas plataformas podem, com o tempo, desestimular a atitude para o consumo colaborativo do indivíduo.

\section{Conclusão}

Esta pesquisa permitiu traçar um panorama abrangente acerca da ampliação do conhecimento científico sobre a influência da consciência ambiental na atitude para o consumo colaborativo de compra, venda e troca em plataformas digitais, como Mercado Livre, OLX, entre outras plataformas. Adicionalmente, caracterizou-se o perfil sociodemográfico desses usuários, e isso tem impacto para as pesquisas que alinham a consciência ambiental ao consumo colaborativo. Para essa abordagem, foi realizada uma pesquisa empírica com 125 estudantes do curso de Administração de uma Universidade Pública.

Em relação à influência da consciência ambiental na atitude para o consumo colaborativo de compra, venda e troca em plataformas digitais, por meio da regressão logística, foram identificadas influências positivas da preocupação com o lixo e da renda individual na atitude para o consumo colaborativo dos universitários. Em contrapartida, a média do uso das plataformas digitais e o semestre tiveram influências negativas na atitude para o consumo colaborativo de compra, venda e troca em plataformas digitais, mostrando que alunos com maior maturidade tendem a reduzir sua participação nessa forma de consumo. Isso permitiu inferir a influência da consciência ambiental, sobretudo pela preocupação com o lixo, na atitude para o consumo colaborativo, o que confirmou a hipótese da pesquisa $\mathbf{H}_{1}$ : Há influência positiva da consciência ambiental na atitude para o consumo colaborativo dos universitários.

Em relação à caracterização dos usuários, por meio de estatística descritiva e inferencial, observou-se que a maior parte da amostra conhece o conceito de consumo 
colaborativo e que quanto mais familiarizada com essa temática, maior a frequência e o tempo médio de uso das plataformas digitais. Destacou-se, ainda, que as plataformas digitais mais operadas pelos universitários são OLX e Mercado Livre e que as principais motivações para uso são redução de custos, redução do tempo de transação e conveniência e facilidade, independente da familiaridade com o conceito. Logo, prevaleceram benefícios individuais em detrimento dos coletivos, como o consumo consciente e o bem-estar da sociedade.

Evidenciou-se também que, apesar das finalidades associadas ao consumo sustentável não terem sido predominantes nesta pesquisa, os indivíduos que praticam a compra, venda ou troca em plataformas digitais estão realizando uma forma de consumo menos danosa ao meio ambiente, visto que essas transações aumentam o tempo de vida útil dos objetos, diminuindo o descarte e a compra de produtos novos e, consequentemente, a eliminação de subprodutos

Diante dos resultados encontrados, esta pesquisa busca ampliar a compreensão da influência da consciência ambiental na atitude para o consumo colaborativo dos universitários, o que contribui diretamente para o desenvolvimento do conhecimento científico no campo do consumo colaborativo e da consciência ambiental. Ademais, destacouse o entendimento de que, apesar de estarem satisfazendo principalmente motivações pessoais, os universitários que realizaram o consumo colaborativo, de forma consciente ou não, podem estar contribuindo para um modelo de consumo mais alinhado ao desenvolvimento sustentável, o que confere uma contribuição para o campo do consumo colaborativo, fenômeno amplo, complexo e objeto de múltiplas discussões e enfoques.

Como implicação gerencial e social, indica-se a possibilidade de ações conjuntas do governo e das empresas, atores responsáveis pela consolidação de uma economia mais sustentável, no sentido do desenvolvimento de políticas públicas que possam estimular a consciência ambiental dos indivíduos. Dessa forma, por meio do poder normativo e comunicativo do Estado, poder-se-ia influenciar o comportamento do consumidor de forma mais imediata, estimulando, a curto prazo, uma forma equilibrada de consumo colaborativo por meio de apelos individuais. Com isso, a longo prazo, o governo poderia estar contribuindo significativamente para a mudança de cultura e conscientização em relação ao consumo, fatores essenciais para uma economia colaborativa mais justa e fortalecida.

Cumpre ressaltar que esta pesquisa se defrontou com algumas limitações, como a realização do estudo com universitários em apenas uma única universidade e em uma única cidade do Brasil, e ainda em uma perspectiva transversal, sem realizar abordagens longitudinais, isto é, comparando as atitudes para o consumo colaborativo desses estudantes em um determinado período de tempo.

Revista Gestão e Secretariado (GeSec), São Paulo, SP, 11(2), maio/ago., 2020, p. 25-49. 
Sugere-se, portanto, para futuras pesquisas, compreender outras formas de consumo colaborativo, bem como suas motivações e relações com outros aspectos. Outrossim, poderiam ser verificados quais fatores influenciam na redução da atitude após a realização de compra, venda ou troca nas plataformas digitais, bem como identificar as diferenças entre os indivíduos de acordo com o seu perfil mais frequente (comprador, vendedor ou trocador de produtos usados) a fim de ampliar o campo do conhecimento científico sobre a consciência ambiental e o consumo colaborativo.

\section{Referências}

Aigrain, P. (2012). Sharing: Culture and the economy in the internet age (p. 244). Amsterdam University Press.

Afonso, T., Zanon, M. Â. G., Locatelli, R. L., \& Afonso, B. P. D. (2016). Consciência ambiental, comportamento pró-ambiental e qualidade de gerenciamento de resíduos em serviços de saúde. Revista de Gestão Ambiental e Sustentabilidade-GeAS, 5(3), 106-119.

Arruda, H. R., Bandeira, E. L., Silva, Á. L. L., \& Rebouças, S. M. D. P. (2016). Consumo Colaborativo e Valores Pessoais: O Caso da Bicicleta Compartilhada. REMark: Revista Brasileira de Marketing, 15(5), 683-698.

Barcellos, M. D. D., \& Silva, C. S. D. (2014). Consumo Colaborativo em Porto Alegre: Vamos de Bike Poa. Vii Encontro Nacional de Estudos do Consumo, Rio de Janeiro, 24.

Barnes, S. J., \& Mattsson, J. (2016). Understanding current and future issues in collaborative consumption: A four-stage Delphi study. Technological Forecasting and Social Change, 104, 200-211.

Bedante, G. N., \& Slongo, L. A. (2004). O comportamento de consumo sustentável e suas relações com a consciência ambiental e a intenção de compra de produtos ecologicamente embalados. Porto Alegre.

Belk, R. (2014). You are what you can access: Sharing and collaborative consumption online. Journal of Business Research, 67(8), 1595-1600.

Benjaafar, S., Kong, G., Li, X., \& Courcoubetis, C. (2018). Peer-to-Peer Product Sharing: Implications for Ownership, Usage, and Social Welfare in the Sharing Economy. Management Science.

Black, I. R., \& Cherrier, H. (2010). Anti-consumption as part of living a sustainable lifestyle: daily practices, contextual motivations and subjective values. Journal of Consumer Behaviour, 9(6), 437-453. 
Botsman, R., \& Rogers, R. (2011). What's mine is yours: how collaborative consumption is changing the way we live. London: Collins.

Castro, B. R. V., Moura, L. R. C., Cunha, N. R. S., \& Pires, R. R. (2016). O Marketing Verde na Tecnologia da Informação: Percepções das Atitudes e Comportamentos dos Profissionais de TI e Ações para Incrementar o Marketing Verde nesse Setor. Revista de Gestão Ambiental e Sustentabilidade-GeAS, 5(1), 1-17.

CNMC (2016). Estudio Sobre Los Nuevos Modelos De Prestacioín De Servicios Y La Economiía Colaborativa-Resultados Preliminares. Comisión Nacional de los Mercados y la Competencia, Madrid, Spain.

Deus, E. G. S. Q., Afonso, B. P. D., \& Afonso, T. (2014). Consciência Ambiental, Atitudes e Intenção de uso das Sacolas Plásticas Não-Recicláveis. Revista de Gestão Ambiental e Sustentabilidade-GeAS, 3(1), 71-87.

Doin, T. A. F (2019). Desvendando a metamorfose da economia do compartilhamento: uma revisão sistemática de literatura para a proposição de um framework integrativo (Doctoral dissertation).

EcoD. Ecodesenvolvimento. (2016). Consumo colaborativo: trocar faz bem. Recuperado em 2 julho, 2017, de http://www.ecodesenvolvimento.org/posts/2016/posts/abril/consumocolaborativo-trocar-faz-bem?tag=consumo_consciente

El-Assi, W., Mahmoud, M. S., \& Habib, K. N. (2017). Effects of built environment and weather on bike sharing demand: a station level analysis of commercial bike sharing in Toronto. Transportation, 44(3), 589-613.

Felson, M., \& Spaeth, J. L. (1978). Community structure and collaborative consumption: A routine activity approach. American behavioral scientist, 21(4), 614-624.

Fraanje, W., \& Spaargaren, G. (2019). What future for collaborative consumption? A practice theoretical account. Journal of cleaner production, 208, 499-508.

Freitas, C. S., Petrini, M. C., \& Silveira, L. M. (2016, September). Desvendando o consumo colaborativo: Uma proposta de tipologia. In CLAV 2016.

Gerwe, O., \& Silva, R. (2020). Clarifying the sharing economy: Conceptualization, typology, antecedents, and effects. Academy of Management Perspectives, 34(1), 65-96.

Giudici, G., Guerini, M., \& Rossi-Lamastra, C. (2019). The creation of cleantech startups at the local level: the role of knowledge availability and environmental awareness. Small Business Economics, 52(4), 815-830. 
Gonçalves-Dias, S. L. F., Teodósio, A. D. S. S., Carvalho, S., \& Silva, H. M. R. (2009). Consciência ambiental: um estudo exploratório sobre suas implicações para o ensino de administração. RAE-eletrônica, 8(1), 1-22.

Gmelin, H., \& Seuring, S. (2014). Determinants of a sustainable new product development. Journal of Cleaner production, 69, 1-9.

Griggs, D., Stafford-Smith, M., Gaffney, O., Rockström, J., Öhman, M. C., Shyamsundar, P., ... \& Noble, I. (2013). Policy: Sustainable development goals for people and planet. Nature, 495(7441), 305-307.

Habibi, M. R., Kim, A., \& Laroche, M. (2016). From sharing to exchange: An extended framework of dual modes of collaborative nonownership consumption. Journal of the Association for Consumer Research, 1(2), 277-294.

Habibi, M. R., Davidson, A., \& Laroche, M. (2017). What managers should know about the sharing economy. Business Horizons, 60(1), 113-121.

Hair, J. F., Black, W. C., Babin, B. J., Anderson, R. E., \& Tatham, R. L. (2009). Análise multivariada de dados. Bookman Editora.

Hajli, N. (2015). Social commerce constructs and consumer's intention to buy. International Journal of Information Management, 35(2), 183-191.

Hamari, J., Sjöklint, M., \& Ukkonen, A. (2016). The sharing economy: Why people participate in collaborative consumption. Journal of the Association for Information Science and Technology, 67(9), 2047-2059.

Han, H., \& Kim, Y. (2010). An investigation of green hotel customers' decision formation: Developing an extended model of the theory of planned behavior. International Journal of Hospitality Management, 29(4), 659-668.

Hedlund, T. (2011). The impact of values, environmental concern, and willingness to accept economic sacrifices to protect the environment on tourists' intentions to buy ecologically sustainable tourism alternatives. Tourism and Hospitality Research, 11(4), 278-288.

Heinrichs, H. (2013). Sharing economy: a potential new pathway to sustainability. GAIAEcological Perspectives for Science and Society, 22(4), 228-231.

Hutchison, E. D. (2018). Dimensions of human behavior: Person and environment. Sage Publications.

Hult, G. (2011). Market-focused sustainability: market orientation plus!. Journal of the Academy of Marketing Science, 39(1), 1-6. 
Jaeger-Erben, M., Rückert-John, J., \& Schäfer, M. (2015). Sustainable consumption through social innovation: a typology of innovations for sustainable consumption practices. Journal of Cleaner Production, 108, 784-798.

Kaiser, F. G., Ranney, M., Hartig, T., \& Bowler, P. A. (1999). Ecological behavior, environmental attitude, and feelings of responsibility for the environment. European psychologist, 4(2), 59-74.

Karahan, E., \& Roehrig, G. (2015). Constructing media artifacts in a social constructivist environment to enhance students' environmental awareness and activism. Journal of Science Education and Technology, 24(1), 103-118.

Karp, D. G. (1996). Values and their effect on pro-environmental behavior. Environment and behavior, 28(1), 111-133.

Kinnear, T. C., Taylor, J. R., \& Ahmed, S. A. (1974). Ecologically concerned consumers: who are they?. The Journal of Marketing, 20-24.

Klineberg, S. L., McKeever, M., \& Rothenbach, B. (1998). Demographic predictors of environmental concern: It does make a difference how it's measured. Social science quarterly, 734-753.

Kuckertz, A., \& Wagner, M. (2010). The influence of sustainability orientation on entrepreneurial intentions-Investigating the role of business experience. Journal of Business Venturing, 25(5), 524-539.

Lam, S. P. (1999). Predicting intentions to conserve water from the theory of planned behavior, perceived moral obligation, and perceived water right. Journal of Applied Social Psychology, 29(5), 1058-1071.

Leismann, K., Schmitt, M., Rohn, H., \& Baedeker, C. (2013). Collaborative consumption: towards a resource-saving consumption culture. Resources, 2(3), 184-203.

Market Analysis (2015). O consumo colaborativo e o consumidor brasileiro. Recuperado em 2 maio, 2020, de http://marketanalysis.com.br/wp-content/uploads/2017/04/2015-MarketAnalysis-O-consumo-colaborativo-e-o-consumidor-brasileiro.pdf

Martin, C. J. (2016). The sharing economy: A pathway to sustainability or a nightmarish form of neoliberal capitalism? Ecological Economics, 121, 149-159.

Maurer, A., Schmitt P. F. Campos, A. P., Silva, S. S. V., \& Barcellos, M. D. (2015). Yes, We also can! O desenvolvimento de iniciativas de consumo colaborativo no Brasil. Revista Base (Administração e Contabilidade) da UNISINOS, 12(1), 69-80.

Morozov, E. (2013). To save everything, click here: The folly of technological solutionism. Public Affairs. 
Morrison, P. S., \& Beer, B. (2017). Consumption and Environmental Awareness: Demographics of the European Experience. In Socioeconomic Environmental Policies and Evaluations in Regional Science (pp. 81-102). Springer Singapore.

Ornellas, R. (2013). Impactos do consumo colaborativo de veículos elétricos na cidade de São Paulo. Future Studies Research Journal, 5(1), 33-62.

Oskamp, S. (2000). A sustainable future for humanity? How can psychology help? American Psychologist, 55(5), 496-508.

Paço, A. M. F., Ferreira, J. M., Raposo, M., Rodrigues, R. G., \& Dinis, A. (2011). Behaviours and entrepreneurial intention: Empirical findings about secondary students. Journal of International Entrepreneurship, 9(1), 20-38.

Paiva, L. E. B., Lima, T. C. B., Sousa, E. S., \& Pontes, E. S. (2019). Sustainability, Innovation, and Entrepreneurship: A Research with Administration and Secretariat Students. Revista de Negócios, 24(3), 7-26.

Pato, C., \& Tamayo, Á. (2006). Valores, creencias ambientales y comportamiento ecológico de activismo. Medio ambiente y comportamiento humano, 7(1), 51-66.

Patricio, L. C. B., \& Kruszielski, L. (2016). Dia de Bicicleta ao Trabalho: Uma Potencial Ferramenta para Planejamento e Promoção da Mobilidade Sustentável. Revista de Gestão Ambiental e Sustentabilidade-GeAS, 5(3), 135-151.

Price, J. A. (1975). Sharing: The integration of intimate economies. Anthropologica, 3-27.

Research Designed For Strategy. (2016). Economia Colaborativa: mudança de cultura ou efeito da crise? Abr. 2016.

Rio+20. Conferência das nações unidas sobre desenvolvimento sustentável. Sobre a conferência. Recuperado em 12 abril, 2017, de http://www.rio20.gov.br/sobre_a_rio_mais_20/desenvolvimento-sustentavel.html

Rivera, J., Gordo, Á., Cassidy, P., \& Apesteguía, A. (2017). A netnographic study of P2P collaborative consumption platforms' user interface and design. Environmental Innovation and Societal Transitions, 23, 11-27.

Santoso, A. S., \& Erdaka, A. (2015). Customer loyalty in collaborative consumption model: Empirical study of CRM for product-service system-based e-commerce in Indonesia. Procedia computer science, 72(1), 543-551.

Schlegelmilch, B. B., Bohlen, G. M., \& Diamantopoulos, A. (1996). The link between green purchasing decisions and measures of environmental consciousness. European journal of marketing, 30(5), 35-55. 
Schmuck, P., \& Schultz, P. W. (2002). Sustainable development as a challenge for psychology. In Psychology of sustainable development (pp. 3-17). Springer US.

Schor, J. B. (2014). Born to buy: the commercialized child and the new consumer cult. Simon and Schuster.

Silva, L. F., Cunto, G. C., \& Guevara, A. J. H. (2016). Consumidor ou Cidadão: Um Estudo Sobre as Externalidades Negativas no Consumo de Energia. Revista de Gestão Ambiental e Sustentabilidade, 5(2), 130-145.

Straughan, R. D., \& Roberts, J. A. (1999). Environmental segmentation alternatives: a look at green consumer behavior in the new millennium. Journal of consumer marketing, 16(6), 558-575.

Thakur, R. (2016). The United Nations, peace and security: from collective security to the responsibility to protect. Cambridge University Press.

Tomas, R. N., Meschgrahw, R. P., \& Alcantara, R. L. C. (2012). As Redes Sociais e o Comportamento De Compra Do Consumidor: O Reinado Do" boca-A-Boca" Está De Volta? REMark, 11(2), 120-147

Verdugo, V. C. (2012). The positive psychology of sustainability. Environment, Development and Sustainability, 14(5), 651-666.

Vermeir, I., \& Verbeke, W. (2008). Sustainable food consumption among young adults in Belgium: Theory of planned behaviour and the role of confidence and values. Ecological economics, 64(3), 542-553.

Vining, J., \& Ebreo, A. (1992). Predicting recycling behavior from global and specific environmental attitudes and changes in recycling opportunities. Journal of applied social psychology, 22(20), 1580-1607.

Young, W., \& Tilley, F. (2006). Can businesses move beyond efficiency? The shift toward effectiveness and equity in the corporate sustainability debate. Business Strategy and the Environment, 15(6), 402-415.

Zervas, G., Proserpio, D., \& Byers, J. W. (2017). The rise of the sharing economy: Estimating the impact of Airbnb on the hotel industry. Journal of Marketing Research, 54(5), 687705.

Submetido em: 13.02 .2020

Aceito em: $\quad 06.07 .2020$ 